\title{
Logistic Growth Curve-based Chinese Professional Basketball Clubs Development Research
}

\author{
Yao Fan*
}

Institute of Physical Education, Northeast Normal University, Changchun 130024, Jilin, China

\begin{abstract}
In contemporary society, so as to enhance Chinese basketball level, reform of Chinese basketball professionalization has been constantly deepened, while basketball clubs development is the internal impetus of Chinese basketball undertakings. The paper analyses specific situation of Chinese basketball clubs, and finds that most of Chinese sports clubs are sponsored and constructed by good corporate such as LuLu Group, Liaoning Bus Group, Wing On Company, Wanma Group and others, and they run through sports industry revenue, gate receipts and else; regarding Chinese basketball clubs coaches age, coaching time, experiences and others, it makes investigation and researches, and gets that though Chinese athletes basketball levels have some improvements, faculty is still deficient, which let Chinese basketball athletes technical levels not to be so high, amount of excellent athletes is not more, so China should increase investment in faculty so that propel to Chinese basketball development; by data sheet after logistic curve changing, it gets that the amount of Chinese basketball clubs will constantly increase in future years that provides training fields for Chinese more excellent athletes training and guarantees for their future development.
\end{abstract}

Keywords: Club, coach, logistic growth curve method, professional basketball.

\section{INTRODUCTION}

The advancement and development of Chinese basketball levels cannot do without great support from Chinese professional basketball clubs, below are research achievement that Chinese relative experts obtain in the field.

Li Bing in "Teenagers professional basketball clubs management and operation mechanism research", through analyzing Chinese national conditions, he carried out specific investigation and research on Chinese teenagers professional basketball clubs, found that China had a large population, teenagers that gifted in basketball were also more, Chinese basketball clubs developed teenagers' basketball interests and reinforced their basketball skills through training so that made talent reserve preparations for Chinese basketball development [1]. The article thought that China should further increase investment in Chinese basketball clubs and facilitate their development [2].

Zhao Jinglun in the article "Athletics city's junior high school basketball clubs development status investigation research", by analyzing Chinese high schools basketball clubs development status, he got that athletics city's junior high school basketball clubs development was relatively stable that provided lots of excellent basketball talents for the city, from which more outstanding talents were chosen by national basketball team, and their achievement in international games were particularly prominent.
It indicated that Athletics city's reform of basketball clubs operation and management ways had good results that guaranteed for the city's basketball undertakings greatly development [3].

Zhang Ji-Wen in the article "Beijing Shougang basketball clubs operation management empirical research", she carried on investigation and research on Chinese Shougang basketball clubs development status, and got that Chinese Shougang basketball clubs' operational ways and management concepts had further improved with years' development, the purpose of Shougang clubs construction was to strengthen workers' physical quality, enrich workers spare time life, let workers' life to be more pleasant, only then can improve workers' working efficiency and guarantee for Chinese undertakings greatly development [4].

The paper utilizes logistic growth curve method to establish basketball clubs' amount prediction model, specific estimates and predicts on Chinese basketball undertakings so that propel to Chinese basketball undertakings development.

\section{MODEL ESTABLISHMENT}

To make the paper more convincing, the paper uses mathematical models to further analyze and research on Chinese basketball clubs recent years' operational ways and reformation results, and predicts on Chinese basketball clubs future development direction and operational management mode, puts forward reasonable development direction [5].

\section{Chinese Basketball Clubs' Essential Features}

With the implementation of globalization in economy, politics and culture, Chinese basketball culture carries on experience exchanges with other countries basketball, which 
Table 1. Chinese partial basketball clubs situations.

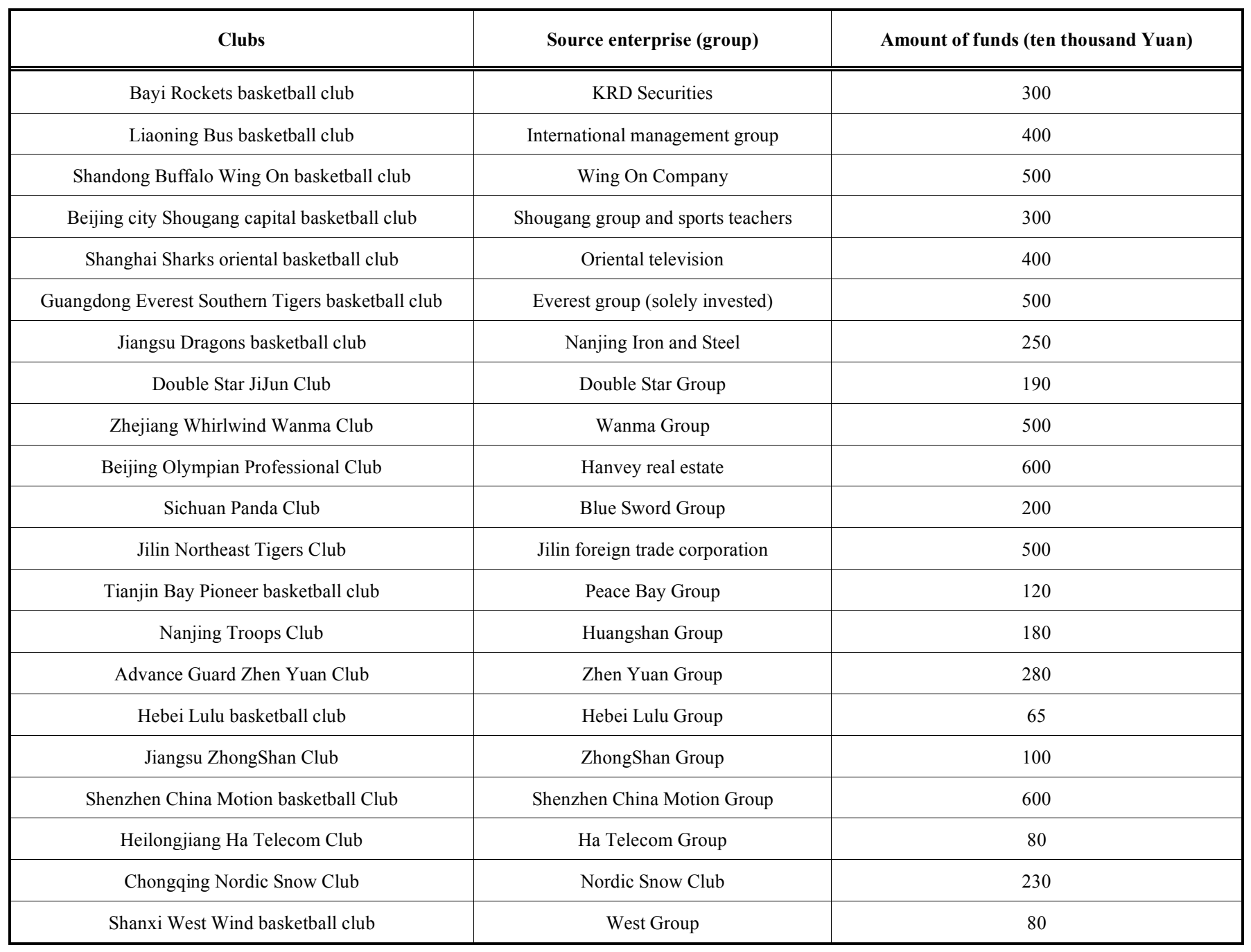

Table 2. Chinese professional basketball undertaken municipal worker average income situation.

\begin{tabular}{|c|c|c|c|}
\hline Name of province and city & Worker average income & Name of province and city & Worker average income \\
\hline \hline Beijing & 28852 & Liaoning & 18390 \\
\hline Shanghai & 33938 & Shandong & 18293 \\
\hline Jiangsu & 17489 & Jilin & 12943 \\
\hline Zhejiang & 23824 & Guangdong & 17283 \\
\hline Xinjiang & 11674 & Shanxi & 12948 \\
\hline
\end{tabular}

exerts a profound impacts on Chinese basketball important part basketball clubs development, and lets Chinese clubs operational ways to be greatly changed as Table 1.

Besides, Chinese professional basketball undertaken municipal worker average income situation also has a great impact on basketball clubs operational status [6].

Below Tables 2, 3 is Chinese professional basketball undertaken municipal worker average income situation, make statistical analysis on the table.
To further analyze Chinese clubs participants income situation and make analysis of basketball clubs development targeted at Chinese clubs participants income situation.

With the improvement of Chinese living standards, the proportion of Chinese education and cultural entertainment consumption is constantly increasing as Tables $\mathbf{4 , 5}$, which shows Chinese habitants entertainment life is relative colorful and participants' investment in clubs is also constantly increasing. 
Table 3. Chinese clubs main funds source situation.

\begin{tabular}{|c|c|c|c|c|c|c|}
\hline Source & Named fee & $\begin{array}{c}\text { Sports industry } \\
\text { development revenue }\end{array}$ & $\begin{array}{c}\text { Chinese basketball } \\
\text { association }\end{array}$ & $\begin{array}{c}\text { Company } \\
\text { investment }\end{array}$ & $\begin{array}{c}\text { Ticket sales } \\
\text { revenue }\end{array}$ & $\begin{array}{c}\text { Advertising } \\
\text { Revenue }\end{array}$ \\
\hline \hline Amount & $\begin{array}{c}700 \text { ten thou- } \\
\text { sand }\end{array}$ & 1000 ten thousand & 200 ten thousand & 570 ten thousand & 300 ten thousand & 25 ten thousand \\
\hline Proportion & $21 \%$ & $27 \%$ & $18 \%$ & $17 \%$ & $15 \%$ & $2 \%$ \\
\hline
\end{tabular}

Table 4. Chinese clubs participants' income situation.

\begin{tabular}{|c|c|c|c|c|}
\hline Year & 2011 & 2012 & 2013 & 2014 \\
\hline Growth rate & $24.1 \%$ & $22.6 \%$ & $29.9 \%$ & $42.3 \%$ \\
\hline
\end{tabular}

Table 5. Chinese education and cultural entertainment services consumption proportion.

\begin{tabular}{|c|c|c|c|c|c|c|}
\hline Source & $\mathbf{2 0 0 9}$ & $\mathbf{2 0 1 0}$ & $\mathbf{2 0 1 1}$ & $\mathbf{2 0 1 2}$ & $\mathbf{2 0 1 3}$ & $\mathbf{2 0 1 4}$ \\
\hline \hline Proportion (\%) & $25.32 \%$ & $25.98 \%$ & $26.43 \%$ & $27.33 \%$ & $27.68 \%$ & $28.12 \%$ \\
\hline
\end{tabular}

\section{Chinese Coaches Situation Investigation}

Chinese coaches are main factors that affect Chinese basketball clubs development, so it makes specific research on Chinese coaches' coaching time, athletes' performance situation, relevant data is as Tables 6-9.

By interviewing and investigating on Chinese large and medium-sized cities basketball clubs' coaches situation, it points out that Chinese clubs coaches' coaching time and experiences are fewer, coaches are around 40 years old that have certain experience, coaches are relative satisfied with equipment evaluation, which is the advancement of clubs management mode.

\section{Logistic Growth Curve-based Sports Systematic Institu- tion Population Prediction}

By Logistic growth curve method, it predicts amount of Chinese basketball clubs during 2015 to 2019, and then points out directions for Chinese clubs development.

\section{Logistic Growth Curve Guiding Thoughts}

Logistic curve general mathematical model is:

$$
\begin{aligned}
& \frac{d y}{d t}=r y\left(1-\frac{y}{L}\right) \\
& y=\frac{L}{1+c e^{-r t}}
\end{aligned}
$$

In the following, it records Logistic curve general form as:

$$
y_{t}=\frac{1}{K+a b^{t}}, K>0, a>0,0<b \neq 1
$$

Among them, in Logistic curve, for parameter estimation, it makes following changes: $y_{t}^{\prime}=\frac{1}{y_{t}}$, that is:

$y_{t}^{\prime}=K+a b^{t}$

For time sequence $n$ pieces of observation values, it average divides them into three parts, and for every part, it has $m$ periods, and then it has $n=3 m$.

Part one: $y_{1}, y_{2}, y_{3}, \cdots, y_{m}$;

Part two: $y_{m+1}, y_{m+2}, y_{m+3}, \cdots, y_{2 m}$;

Part three: $y_{2 m+1}, y_{2 m+2}, y_{2 m+3}, \cdots, y_{3 m}$

Among them, every part trend sum is equal to corresponding observation values sum, therefore provide parameters estimation, three sums method steps are as following:

Record observation values each part sum is:

$S_{1}=\sum_{t=1}^{m} y_{t}^{\prime}, S_{2}=\sum_{t=m+1}^{2 m} y_{t}^{\prime}, S_{3}=\sum_{t=2 m+1}^{3 m} y_{t}^{\prime}$

And it has:

$\left\{\begin{array}{l}S_{1}=\sum_{t=1}^{m} \hat{y}_{t}=\sum_{t=1}^{m}\left(K+a b^{t}\right)=m K+a b\left(1+b+b^{2}+\cdots+b^{m-1}\right) \\ S_{2}=\sum_{t=m+1}^{2 m} \hat{y}_{t}=\sum_{t=m+1}^{2 m}\left(K+a b^{t}\right)=m K+a b^{m+1}\left(1+b+b^{2}+\cdots+b^{m-1}\right) \\ S_{3}=\sum_{t=2 m+1}^{3 m} \hat{y}_{t}=\sum_{t=2 m+1}^{3 m}\left(K+a b^{t}\right)=m K+a b^{2 m+1}\left(1+b+b^{2}+\cdots+b^{m-1}\right)\end{array}\right.$

Among them: $\left(1+b+b^{2}+\cdots+b^{m-1}\right)(b-1)=b^{m}-1$ 
Table 6. Chinese coaches coaching time.

\begin{tabular}{|c|c|c|c|c|c|}
\hline Coaching time & 4 years & 5-8 years & 9-12 years & 13-16 years & $17-20$ years \\
\hline Number of people & 3 & 3 & 2 & 6 & 1 \\
\hline
\end{tabular}

Table 7. Sports performance situation when coaches teach athletes.

\begin{tabular}{|c|c|c|}
\hline Performance situation & Frequency & Percentage \\
\hline \hline Master sportsman in international level & 1 & $6 \%$ \\
\hline Master sportsman in national level & 12 & $81 \%$ \\
\hline National first grade sportsman & 2 & $13 \%$ \\
\hline National second grade sportsman & 0 & 0 \\
\hline
\end{tabular}

Table 8. Coaches' age group.

\begin{tabular}{|c|c|c|c|c|c|}
\hline Age & $\mathbf{2 6 - 3 0}$ years old & $\mathbf{3 1 - 3 5}$ years old & $\mathbf{3 6 - 4 0}$ years old & 41-45 years old & 46-50 years old \\
\hline \hline Frequency & 28 & 46 & 43 & 84 & 28 \\
\hline Proportion & $7 \%$ & $21 \%$ & $20 \%$ & $39 \%$ & $7 \%$ \\
\hline
\end{tabular}

Table 9. Coaches team members setting satisfaction.

\begin{tabular}{|c|c|c|c|c|}
\hline Attitude & Very satisfied & Satisfied & Basic satisfied & Not satisfied \\
\hline \hline Frequency & 6 & 7 & 2 & 0 \\
\hline Proportion & $40 \%$ & $47 \%$ & $13 \%$ & 0 \\
\hline
\end{tabular}

And then it can get:

$$
\left\{\begin{array}{l}
S_{1}=m K+a b \frac{b^{m-1}}{b-1} \\
S_{2}=m K+a b^{m+1} \frac{b^{m-1}}{b-1} \\
S_{3}=m K+a b^{2 m+1} \frac{b^{m-1}}{b-1}
\end{array}\right.
$$

Therefore, it can get:

$$
\left\{\begin{array}{l}
b=\left(\frac{S_{3}-S_{2}}{S_{2}-S_{1}}\right)^{\frac{1}{m}} \\
a=\left(S_{2}-S_{1}\right) \frac{b-1}{b\left(b^{m}-1\right)^{2}} \\
K=\frac{1}{m}\left[S_{1}-\frac{a b\left(b^{m}-1\right)}{(b-1)}\right]
\end{array}\right.
$$

Besides, when predict data, it should test data, test method is:

$$
\frac{y_{t+1}-y_{t}}{y_{t}-y_{t-1}} \approx b
$$

Logistic curve data handling and conclusions

By

$y_{t}^{\prime}=\frac{1}{y_{t}}$,

it gets year 2008 2012 data after changing is as following Table 10:

According to formula(5), it gets:

$S_{1}^{1}=0.682, S_{2}^{1}=0.614, S_{3}^{1}=0.478$

$S_{1}^{2}=0.1274, S_{2}^{2}=0.1256, S_{3}^{2}=0.1232$

$S_{1}^{3}=0.0778, S_{2}^{3}=0.0817, S_{3}^{3}=0.0766$

Then according to formula (8), it gets:

$b^{1}=1.867, a^{1}=-0.005111, K^{1}=0.35468$

$b^{2}=1.6999, a^{2}=-0.0001453, K^{2}=0.1281$ 
Table 10. Data sheet after logistic curve changing.

\begin{tabular}{|c|c|c|c|c|c|c|c|}
\hline \multirow{3}{*}{$y_{t}^{\prime} / \times 10^{3}$} & Year & $\mathbf{2 0 0 9}$ & $\mathbf{2 0 1 0}$ & $\mathbf{2 0 1 1}$ & $\mathbf{2 0 1 2}$ & $\mathbf{2 0 1 3}$ & $\mathbf{2 0 1 4}$ \\
\cline { 2 - 8 } & Professional basketball club & 0.578 & 0.447 & 0.437 & 0.477 & 0.244 & 0.163 \\
\cline { 2 - 8 } & Teenager basketball club & 0.0841 & 0.0533 & 0.0330 & 0.0537 & 0.0591 & 0.0613 \\
\cline { 2 - 8 } & Amateur basketball club & 0.0378 & 0.0365 & 0.0567 & 0.0612 & 0.0414 & 0.0462 \\
\hline
\end{tabular}

Table 11. Prediction on amount of Chinese basketball clubs.

\begin{tabular}{|c|c|c|c|c|c|}
\hline & 2015 & 2016 & 2017 & 2018 & 2019 \\
\hline Professional basketball club & 67 & 75 & 77 & 79 & 86 \\
\hline Amateur basketball club & 116 & 136 & 144 & 168 & 186 \\
\hline
\end{tabular}

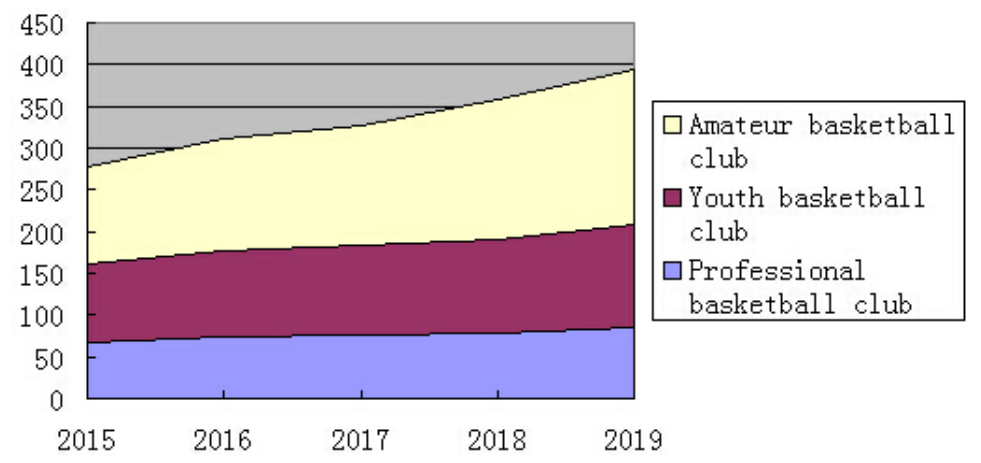

Fig. (1). The number of basketball clubs in China.

$b^{3}=1.5128, a^{3}=0.000786, K^{3}=0.0758$

So obtained sports systematic institution population's logistic growth curve mathematical model is:

$$
\left\{\begin{array}{l}
y_{t}^{1}=\frac{1}{0.35468-0.00511 \times 1.867^{t}} \\
y_{t}^{2}=\frac{1}{0.1281-0.0001453 \times 1.6999^{t}} \\
y_{t}^{3}=\frac{1}{0.0748+0.000796 \times 1.5128^{t}}
\end{array}\right.
$$

When predicting on Chinese sports systematic institution population development changes in future five years after 2012 , only need to input $t$ value into above formula, as predict $y_{2013}$, then it has $t=2013-2004+1=10$.And then it can get following Table 11 predicted result:

Draw above predicted population into following broken line Fig. (1), it better analyzes sports systematic institution population trend:

By above broken line statistical Fig. (1) analysis, it gets conclusion that in five years after 2014, the amount of national different types basketball clubs will be sharply increased, which let Chinese basketball undertakings to be rapidly developed, from which players techniques would be further strengthened that makes preparations for China great deals of lead players training.

\section{CONCLUSION}

The paper firstly makes specific research and analysis of Chinese basketball clubs distribution, income situation, it gets that Chinese basketball clubs are mainly set up by large enterprises sponsorship, and they maintain clubs normal operation through sports industry revenue, gate receipts, named fee, games and other incomes. Secondly, the paper researches on Chinese coaches' situations, it gets that Chinese coaches' coaching time is shorter; experiences are deficient that hinders Chinese basketball clubs development. Chinese should increase investment in Chinese basketball faculty so that let Chinese basketball clubs to run normally. Finally, by data sheet after logistic curve changing, it gets that amount of basketball clubs is constantly increasing, nation and enterprises investment increase, which offers commitment and support for Chinese basketball development.

\section{CONFLICT OF INTEREST}

The author confirms that this article content has no conflict of interest.

\section{ACKNOWLEDGEMENTS}

Declared none. 


\section{REFERENCES}

[1] J. Chen, and S. Yao, "Application of connected analysis to analysis of statistical data of basketball technique," Journal of Shanghai Physical Education Institute, vol. 33, no. 5, pp. 91-94, 2009.

[2] F. Fu, Z. Yu, and Q. Zhang, "Analysis of offensive turnover in period of men's basketball final matches at $10^{\text {th }}$ National Games," Shandong Sports Science \& Technology, vol. 28, no. 2, pp. 24-25, 2006.

[3] J. Li, "Research on the current situation and developing strategy of chinese track and field coaches," Journal of Shenyang Sport University, vol. 25 , no. 2 , pp. 63-66, 2006.
[4] J. Mao, and S. Shan, "Basketball technology statistical software development," Journal of Wuhan Institute of Physical Education, vol. 46, no. 2, pp. 70-73, 2012.

[5] Y. Yang, H. Rao, and J. Len, "A study on the improvement of computer statistic software for basketball games," Journal of Hubei Sports Science, vol. 22, no. 2, pp. 204-205, 2003.

[6] L. Zhang, "Analysis on foul in man's basketball final phase in $10^{\text {th }}$ national games," China Sport Science and Technology, vol. 42, no. 1 , pp. 50-52, 2006

Received: May 26, 2015

Revised: July 14, 2015

Accepted: August 10, 2015

(C) Yao Fan; Licensee Bentham Open.

This is an open access article licensed under the terms of the (https://creativecommons.org/licenses/by/4.0/legalcode), which permits unrestricted, noncommercial use, distribution and reproduction in any medium, provided the work is properly cited. 